Jan-Olav Henriksen

\title{
Religion as Orientation and Transformation
}

\author{
A Maximalist Theory \\ [Religion als Orientierung und Transformation. Eine maximalistische Theorie.]
}

Veröffentlicht auf Englisch.

In diesem Buch präsentiert Jan-Olav Henriksen Argumente für das Verständnis von Religion als Ausdruck verschiedener Praktiken: der der Orientierung, der Transformation und der Reflexion. Anstatt Religion in erster Linie auf der Grundlage von Lehre und propositional artikulierten Glaubenssätzen zu verstehen, argumentiert er, dass Religionen in erster Linie als Praxis verstanden werden sollte, die symbolische Ressourcen für Orientierung und Transformation vermittelt. Die Bedeutung von Lehre und Reflexion wird durch ihre Beziehung zu dieser Praxis konstituiert. So bildet die Lehre nicht die Religion. Dieser Ansatz ermöglicht ein maximalistisches Verständnis der Religion, d. h. Religionen als eine Vielfalt von Phänomenen, die alle Dimensionen menschlicher Erfahrung betreffen, zu sehen. Dies ist aus einer reduktionistischen Perspektive nicht möglich. Der Band liefert ein konkretes, praxisorientiertes und pragmatistisches Verständnis der Rolle von Religion in verschiedenen Bereichen des menschlichen Lebens.

Jan-Olav Henriksen Born 1961; 1990 Dr. theol.; 2000 Alan Richardson Fellow at the University of Durham, UK; 2002 Dr. philos.; since 1994 professor of systematic theology at MF Norwegian School of Theology; currently also Dean of Research; member of the Center of Theological Inquiry, Princeton, NJ.

\section{Jetzt bestellen:}

https://mohrsiebeck.com/buch/religion-as-orientation-and-transformation-9783161550997?no_cache=1 order@mohrsiebeck.com

Telefon: $+49(0) 7071-923-17$

Telefax: $+49(0) 7071-51104$ 